\title{
Unified Versus Divided Enrollment in School Choice: Improving Student Welfare in Chicago
}

\author{
Battal Doğan \\ M. Bumin Yenmez
}

Discussion Paper 18 / 705

18 November 2018

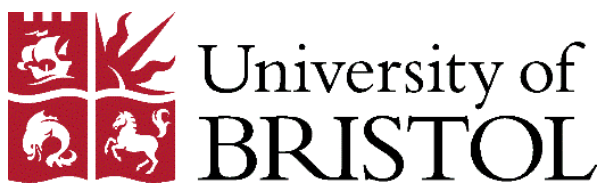

Department of Economics

University of Bristol

Priory Road Complex

Bristol BS8 1TU

United Kingdom 


\title{
UNIFIED VERSUS DIVIDED ENROLLMENT IN SCHOOL CHOICE: IMPROVING STUDENT WELFARE IN CHICAGO
}

\author{
BATTAL DOĞAN AND M. BUMIN YENMEZ
}

\begin{abstract}
The Chicago Board of Education is implementing a centralized clearinghouse to assign students to schools for 2018-19 admissions. In this clearinghouse, each student can simultaneously be admitted to a selective and a nonselective school. We study this divided enrollment system and show that an alternative unified enrollment system, which elicits the preferences of students over all schools and assigns each student to only one school, is better for students when choice rules of schools are substitutable. Furthermore, we characterize the sources of inefficiency in the divided system.
\end{abstract}

\section{Introduction}

Market design has been especially successful in assigning students to public schools. In an influential study, Abdulkadiroğlu and Sönmez (2003) have introduced market design as a tool in this context, shown that some school districts use deficient mechanisms, and proposed better alternatives. Since then many school districts including Boston, Denver, Detroit, New Orleans, New York City, Newark, and Washington D.C. have either established centralized clearinghouses or reformed their existing systems. ${ }^{1}$

In April 2017, the Chicago Board of Education voted unanimously to implement a centralized clearinghouse to assign students to schools. Some of these schools are called 'selective' and use standardized testing for admissions, while the rest are 'nonselective,' district-run public schools that may include magnet and neighborhood schools. Prior to April 2017, the admissions to selective schools were centralized whereas the admissions

Date: June 18, 2018.

Keywords: Market design, school choice, unified enrollment.

Doğan is affiliated with Faculty of Business and Economics (HEC), University of Lausanne, 1015 Lausanne, Switzerland; Yenmez is with Department of Economics, Boston College, 140 Commonwealth Ave, Chestnut Hill, MA, 02467. Emails: battal.dogan@unil.ch, bumin. yenmez@bc.edu. We thank Lars Ehlers, Bettina Klaus, Scott Kominers, Alexey Kushnir, Vikram Manjunath, William Thomson and participants at several seminars and conferences for comments. Battal Doğan gratefully acknowledges financial support from the Swiss National Science Foundation (SNSF) and the hospitality of the Center of Mathematical Sciences and Applications (CMSA, Harvard University) where part of this paper was written. This paper has been circulated as a part of our previous working paper entitled "How to improve student assignment in Chicago: unified enrollment in school choice," which is obsolete now.

${ }^{1}$ See Abdulkadiroğlu et al. (2005a) and Abdulkadiroğlu et al. (2005b) for the reforms in Boston and New York City, respectively. 
to nonselective schools were not. In the impending system, each student is admitted to a selective and a nonselective school. We call this the divided enrollment system because a student can be assigned to more than one school in the clearinghouse. Instead, we propose a unified enrollment system in which each student can be assigned to only one school.

We study divided and unified enrollment systems for student assignment when there are different types of schools; we show that the unified enrollment system is better for students than the divided one when choice rules of schools are substitutable which requires that a student admitted from a set is also admitted from any subset including her. Therefore, we show that the impending system in Chicago can be improved for students by moving to the unified enrollment system. Furthermore, the unified system can be established easily, using the existing mechanisms, with only one change: Instead of eliciting the preferences of students over selective and nonselective schools separately, students' preferences over all schools are elicited.

Throughout the paper, we assume that the student-proposing deferred acceptance algorithm (SPDA) of Gale and Shapley (1962) is used to assign students to schools in the unified enrollment system or for each type of school in the divided enrollment system. As far as we know, all of the aforementioned school districts currently use this algorithm. We show that either the outcome of the unified enrollment system Pareto dominates the outcome of the divided enrollment system or they are the same when choice rules are substitutable (Theorem 1). When there exists one school with a choice rule that is not substitutable, we construct student preferences such that at least one student strictly prefers the divided enrollment outcome to the unified enrollment outcome (Theorem 2). Therefore, substitutability is a necessary and sufficient condition for the student welfare comparison to hold for all student preferences between the two enrollment systems.

The fact that the unification of the enrollment system brings about efficiency gains is in line with prior market-design experiences in which centralization brings about efficiency gains. Yet, the exact sources of efficiency gains from centralization had been relatively less understood. Here, we also characterize the sources of inefficiency in the divided enrollment system, which provides some insights on the efficiency gains from centralization under substitutable choice rules. We identify three reasons for the inefficiency resulting from a divided enrollment system. One obvious reason is that some of the seats may be wasted, as a student can get into two or more schools but can accept only one. However, the inefficiency of the divided enrollment system is not limited to this waste. A second possible reason of inefficiency is miscoordination. In the divided enrollment system, two students may end up applying to the same pair of schools and block each other from receiving more preferred schools, while the unified enrollment system would have coordinated their preferences and they would not have applied to each other's more preferred schools. A 
third possible reason of inefficiency is a new interrupter. In the divided enrollment system, a student may apply to a school that he would not have applied to in the unified enrollment system, and interrupt the admission process of the school eventually not receiving an offer from that school but hurting some other students. ${ }^{2}$ We show that the entire efficiency loss due to the divided enrollment is characterized by the above three possible reasons (Theorem 3).

Related Literature. An important feature of our model is the coexistence of two centralized enrollment systems involving the same set of students. Manjunath and Turhan (2016) also consider a model with the same feature and show that the divided enrollment system cannot always avoid waste (in their Theorem 1) and cannot always coincide with the unified enrollment outcome (in their Corollary 1). More relatedly, a special case of our Theorem 1 that the unified enrollment Pareto improves over the divided enrollment system can be shown using their Theorem 2. Turhan (2017) studies the mechanism introduced in Manjunath and Turhan (2016) when there are different partitions of schools and analyzes the welfare and incentive implications when the partition becomes finer or coarser.

The college admissions in France features a unified enrollment system with multiple stages. Like the impending system in Chicago there are selective and nonselective schools. For nonselective schools in France, there are no priorities. Bonkoungou (2017) studies different tie-breaking rules in a static setting and shows that there is no strategy-proof tie-breaking rule and further studies the incentive properties of these rules. On the other hand, Haeringer and Iehlé (2017) focus on the multiple-stage feature of the French college admissions. They weaken stability and study mechanisms that satisfy this stability notion. Unlike these two papers, we consider a divided enrollment system and establish that the unified enrollment system is better for students.

\section{Model}

2.1. Primitives. Let $\mathcal{S}=\left\{s_{1}, \ldots, s_{|\mathcal{S}|}\right\}$ be a set of students and $\mathcal{C}=\left\{c_{1}, \ldots, c_{|\mathcal{C}|}\right\}$ be a set of schools. Each school has a type. In our motivating Chicago Public School application, there are two school types: selective and nonselective. In general, there are $K \geq 2$ school types. The set of type- $k$ schools is nonempty and denoted by $\mathcal{C}_{k}$ for each type $k$. Therefore, $\left\{\mathcal{C}_{1}, \ldots, \mathcal{C}_{K}\right\}$ is a partition of the set of schools $\mathcal{C}$.

\footnotetext{
${ }^{2}$ Even in the unified enrollment system, there may be interrupters. The divided enrollment system may bring new interrupters, which is the third source of inefficiency that we are pointing out.
} 
Each student $s \in \mathcal{S}$ has a preference relation $R_{s}$ over $\mathcal{C} \cup\{s\},{ }^{3}$ where $s$ represents an outside option for the student, which can be the neighborhood school, private school, or homeschooling. Given $c, c^{\prime} \in \mathcal{C} \cup\{s\}$, we write $c P_{s} c^{\prime}$ if $c \neq c^{\prime}$ and $c R c^{\prime}$, i.e., student $s$ strictly prefers school $c$ to school $c^{\prime}$. A school $c$ is acceptable to student $s$ if the school is strictly better than the outside option, or equivalently, $c P_{S} s$.

Each school $c \in \mathcal{C}$ has a capacity $q_{c} \in \mathbb{N}$, which represents the maximum number of students the school can admit. The admission policy of school $c$ is represented by a choice rule $C h_{c}: 2^{\mathcal{S}} \rightarrow 2^{\mathcal{S}}$, which maps each nonempty set $S \subseteq \mathcal{S}$ of students to a subset $C h_{c}(S) \subseteq S$ of chosen students such that $\left|C h_{c}(S)\right| \leq q_{c}$. Thus, the school cannot accept more students than its capacity. A student $s \in \mathcal{S}$ is acceptable for school $c$ if there exists $S \subseteq \mathcal{S}$ such that $s \in C h_{c}(S)$.

The following property of choice rules play a critical role in our analysis. Choice rule $C h_{c}$ satisfies substitutability if, $s \in S \subseteq S^{\prime} \subseteq \mathcal{S}$ and $s \in C h_{c}\left(S^{\prime}\right)$ imply $s \in C h_{c}(S)$. Substitutability states that when a student is chosen from a set, then she is also chosen from any subset that includes her.

A market is a tuple $\left(\mathcal{S},\left(\mathcal{C}_{k}\right)_{k \in\{1, \ldots, K\}},\left(R_{s}\right)_{s \in \mathcal{S}},\left(q_{c}, C h_{c}\right)_{c \in \mathcal{C}}\right)$.

2.2. Solution Concepts. A matching $\mu$ is an assignment of students to schools that satisfies the capacity constraints:

- for each student $s, \mu(s) \in \mathcal{C}$ or $\mu(s)=s$,

- for each school $c, \mu(c) \subseteq \mathcal{S}$ and $|\mu(c)| \leq q_{c}$, and

- for each student $s$ and school $c, \mu(s)=c$ if, and only if, $s \in \mu(c)$.

In words, every student is either matched with a school or matched to his outside option (which we also refer to as being unmatched), and every school is assigned a set of students with cardinality less than or equal to its capacity. In addition, there is a feasibility constraint that if a student gets matched with a school, then the student is in the set of students that is assigned to the school.

A matching $\mu$ Pareto dominates another matching $v$, if for every student $s, \mu(s)$ is weakly more preferred than $v(s)$ and for one student it is strictly more preferred. A matching that is not Pareto dominated is called Pareto efficient; otherwise, it is Pareto inefficient.

A matching $\mu$ is stable if it satisfies the following properties:

- (individual rationality for students) for each student $s, \mu(s) R_{s} s$,

- (individual rationality for schools) for each school $c, C h_{c}(\mu(c))=\mu(c)$, and

\footnotetext{
${ }^{3}$ More formally, a preference relation over $\mathcal{C} \cup\{s\}$ is a complete, transitive, and anti-symmetric binary relation over $\mathcal{C} \cup\{s\}$. Binary relation $R_{s}$ over $\mathcal{C} \cup\{s\}$ is complete if, for every $c_{1}, c_{2} \in \mathcal{C} \cup\{s\}, c_{1} R_{s} c_{2}$ or $c_{2} R_{s} c_{1}$. It is transitive if, for every $c_{1}, c_{2}, c_{3} \in \mathcal{C} \cup\{s\}, c_{1} R_{s} c_{2}$ and $c_{2} R_{s} c_{3}$ imply $c_{1} R_{s} c_{3}$. It is anti-symmetric if, for every $c_{1}, c_{2} \in \mathcal{C} \cup\{s\}, c_{1} R_{s} c_{2}$ and $c_{2} R_{s} c_{1}$ imply $c_{1}=c_{2}$.
} 
- (no blocking) there exists no student-school pair $(s, c)$ such that $c P_{s} \mu(s)$ and $s \in C h_{c}(\mu(c) \cup\{s\})$.

Individual rationality for a student means that she weakly prefers the outcome over being unmatched. On the other hand, for a school, it means that the school would like to keep all the students assigned to it. No blocking rules out the existence of a student-school pair such that the student strongly prefers the school more than her match and the school would like to admit the student.

In student-assignment settings, stability of a matching is viewed as a fairness notion (Abdulkadiroğlu and Sönmez, 2003). Furthermore, the deferred-acceptance algorithm of Gale and Shapley (1962) is implemented in school districts that have reformed their student assignment mechanisms. The following describes how the student-proposing deferred acceptance algorithm (SPDA) works for a set of students $S$ and a set of schools C.

Step 1. Each student applies to his top-ranked acceptable school in $C$. If there is no such school, then she is unmatched. Each school $c$ considers its applicants, say $A_{1}(c)$. Among these, it tentatively accepts $C h_{c}\left(A_{1}(c)\right)$. It rejects all other applicants. If there is no rejection by any school at this step, then stop and return $\cup_{c} C h_{c}\left(A_{1}(c)\right)$.

Step $t \geq 2$. Each student who is rejected at Step $t-1$ applies to his topranked acceptable school among the ones that have not rejected him. If there is no such school, she is unmatched. Each school $c$ considers the students that it tentatively accepted at Step $t-1$ and the new applicants at Step $t$, say $A_{t}(c)$. Among these, it tentatively accepts $C h_{c}\left(A_{t}(c)\right)$. It rejects all other applicants. If there is no rejection by any school at this step, then stop and return $\cup_{c} C h_{c}\left(A_{t}(c)\right)$.

The algorithm stops in finite time since there can only be a finite number of rejections. When choice rules satisfy substitutability and a mild consistency condition, the outcome of SPDA is a stable matching. ${ }^{4}$

2.3. Enrollment Systems. The unified enrollment system is used in various school districts in the United States, as well as in college admissions in some countries around the world where students are matched with schools (or colleges) in a centralized clearinghouse. However, even though the proposed student assignment system in Chicago is centralized, it is divided in the sense that each student can be admitted to a selective school and a nonselective school simultaneously.

\footnotetext{
${ }^{4}$ See Aygün and Sönmez (2013) for the consistency condition.
} 
Unified Enrollment System: Each student reports a preference relation over all schools. Then a matching is determined by running SPDA. Therefore, each student is matched to at most one school. Finally, each student either accepts his match or rejects it (in which case he is unmatched or matched to his outside option).

Divided Enrollment System: Each student reports, for each school type, his preference relation over the schools of that type, so he reports $K$ separate preference relations. Then, a matching for each school type is determined by running SPDA. As a result, each student may be matched to one school of every type (in the Chicago Public School application each student may be matched with a selective school and a nonselective school). Finally, each student accepts at most one school from his matches (possibly rejecting all of them).

We assume that each student submits her true preferences over all schools in the unified enrollment system and over schools of the same type in the divided enrollment system. Furthermore, each student accepts the most preferred school that she got into in the divided enrollment system and accepts the offer if she gets one in the unified enrollment system. Accepting the most preferred outcome (including the outside option) is the weakly dominant action for each student. Likewise, reporting the true preferences at SPDA is a weakly dominant strategy when choice rules satisfy substitutability and another condition called the law of aggregate demand (Hatfield and Milgrom, 2005). As a result, under these two conditions, both enrollment systems have a subgame-perfect Nash equilibrium in which students submit true preferences at SPDA and accept the most preferable offer afterwards, which is the outcome that we analyze.

\section{Unified Enrollment vs. Divided Enrollment}

We show that the outcome of the unified enrollment system is weakly better than the outcome of the divided enrollment system for all students when choice rules are substitutable. Intuitively, there is more competition for every school type in the divided enrollment system compared to the unified enrollment system because all students compete to get into schools of a given type even if they end up rejecting their offers from these schools eventually. This intuition is formalized in the following lemma.

Lemma 1. Suppose that choice rules of schools satisfy substitutability. Then the set of students who have applied to a school at or before Step t of SPDA in the unified enrollment system is a subset of the corresponding set for the divided enrollment system, for every $t$.

As a result, each student is weakly worse off in the divided enrollment system than in the unified enrollment system under substitutability. 
Theorem 1. Suppose that choice rules of schools satisfy substitutability. Then every student gets a weakly more preferred outcome in the unified enrollment system compared to the the divided enrollment system.

Theorem 1 shows that the divided enrollment system is weakly Pareto inferior to the unified enrollment system when choice rules of schools are substitutable. It generalizes Theorem 2 in Manjunath and Turhan (2016) since they assume that school choice rules are responsive. ${ }^{5}$ Responsive choice rules satisfy substitutability. ${ }^{6}$ Echenique (2007) shows that substitutable choice rules are exponentially more than responsive choice rules.

The intuition that there is more competition in the divided enrollment compared to the unified enrollment does not hold when choice rules are not substitutable.

Theorem 2. Suppose that the choice rule of a school does not satisfy substitutability. Then there exist student preferences such that at least one student gets a strictly less preferred outcome in the unified enrollment system compared to the divided enrollment system.

When there exists a school with a choice rule that does not satisfy substitutability, a student may be chosen from a set while she is rejected from a subset. As a result, the additional competition for this school may make the student receive a more preferred school. In the proof, we build on this intuition and construct student preferences such that this student receives a strictly more preferred outcome in the divided enrollment system compared to the unified enrollment system.

Next, we formally analyze these sources of inefficiency when choice rules are substitutable and show that the entire efficiency loss due to the divided enrollment is characterized by three possible reasons. For this result, we assume that for each school $c \in \mathcal{C}$, choice rule $\mathrm{Ch}_{c}$ satisfies the following additional property.

Conditional capacity-filling: For each $S \subseteq \mathcal{S}$ and $s \in S \backslash C h_{c}(S)$, either $\left|C h_{c}(S)\right|=q_{c}$ or student $s$ is not acceptable to school $c$.

Conditional capacity-filling requires each school to choose a student whenever there is remaining capacity if the student is acceptable. Choice rules in the current Chicago school choice system, which allow them to achieve diversity, satisfy substitutability and conditional capacity-filling (Echenique and Yenmez, 2015).

Given a market, let $S \subseteq \mathcal{S}$ be the set of students who are matched to different schools at the outcome of the divided enrollment system and the outcome of the unified enrollment system. Note that, students in $S$ are worse off in the divided enrollment system by

\footnotetext{
${ }^{5} \mathrm{~A}$ school choice rule is responsive if it has a priority ranking over students and chooses the best acceptable students up to the capacity. The proof of this special case also follows from Theorem 3.4 in $\mathrm{Wu}$ and Roth (2018).

${ }^{6}$ See Chambers and Yenmez (2017) for a characterization of responsive choice rules using substitutability.
} 
Theorem 1, i.e., they suffer from divided enrollment. Let $\mu^{u}$ denote the outcome of the unified enrollment system and let $\mu^{d}$ denote the outcome of the divided enrollment system.

The first source of inefficiency is due to waste. A student who has a relatively higher priority at both selective and nonselective schools may receive offers from two schools, and a seat at the school that he ends up rejecting may be wasted if there is another student who prefers that school to schools that make him offers. In fact, the waste can hurt a chain of students if the student who loses his seat due to waste ends up taking the seat of another student at another school, and so on. We formalize this below.

Say that a student $s \in S$ suffers from waste if there exists a list of students $\left(s_{1}, \ldots, s_{n}\right)$ such that $s_{1}=s$ and

(1) in the divided enrollment system, each student in the list receives the assignment of the preceding student in unified enrollment school, i.e., for each $i \in\{2, \ldots, n\}$, $\mu^{d}\left(s_{i}\right)=\mu^{u}\left(s_{i-1}\right)$,

(2) for each student in the list except student $s_{n}$, the school that he is assigned to in the unified enrollment system does not have an empty seat in the divided enrollment system, i.e., for each $i \in\{1, \ldots, n-1\}$, if $\mu^{u}\left(s_{i}\right)=c$, then $\left|\mu^{d}(c)\right|=q_{c}$, and

(3) the school that student $s_{n}$ is matched to in the unified enrollment system has an unassigned seat at the divided enrollment system, i.e., if $\mu^{u}\left(s_{n}\right)=c$, then $\left|\mu^{d}(c)\right|<q_{c}$.

The second source of inefficiency is due to miscoordination. Suppose that there are two students $s$ and $s^{\prime}$, and two schools one of which is a selective school and the other a nonselective school. Suppose also that student $s$ prefers the nonselective school and student $s^{\prime}$ prefers the selective school. In the divided enrollment system, they may end up applying to both schools and block each other from receiving more preferred schools, while the unified enrollment system would have coordinated their applications and they would not have applied to each other's more preferred schools. In fact, this type of inefficiency can also happen through a longer cycle of students as follows.

Say that a student $s \in S$ suffers from miscoordination if there exists a list of students $\left(s_{1}, \ldots, s_{n}\right)$ such that $s_{1}=s$ and

(1) in the divided enrollment system, each student in the list receives the school of the preceding student in the unified enrollment system, i.e., for each $i \in\{2, \ldots, n\}$, $\mu^{d}\left(s_{i}\right)=\mu^{u}\left(s_{i-1}\right)$, and student $s_{1}$ receives the school that student $s_{n}$ receives in the unified enrollment system, that is, $\mu^{d}\left(s_{1}\right)=\mu^{u}\left(s_{n}\right)$,

(2) for each student the school that she receives in the unified enrollment system does not have an empty seat in the divided enrollment outcome, i.e., for each $i \in\{1, \ldots, n\}$, if $\mu^{u}\left(s_{i}\right)=c$ then $\left|\mu^{d}(c)\right|=q_{c}$, and 
(3) there exists a student in the list whose unified enrollment school has a different type than the type of the unified enrollment school of another student in the list.

The third source of inefficiency is due to a new interrupter. In the divided enrollment system, a student may apply to a school that he would not have applied to in the unified enrollment system, and interrupt the admission process of the school eventually not receiving an offer from that school but hurting some other students.

Say that student $s$ is an interrupter for student $s^{\prime}$ at school $c$ if there is a step at SPDA where student $s$ is tentatively accepted by school $c$ and student $s^{\prime}$ is rejected, while at a later step student $s$ is also rejected (Kesten, 2010). Whenever such a student $s^{\prime}$ exists, we also simply say that student $s$ is an interrupter at school $c$. Note that according to this definition, student $s$ could be accepted by the school for the first time at an earlier step without rejecting any student, but the school may reject another student at a later step while still accepting student $s$. In this case, student $s$ would still be an interrupter if she is rejected at a later step.

Say that a student $s \in S$ suffers from a new interrupter if there exists a list of students $\left(s_{1}, \ldots, s_{n}\right)$ and a student $s^{*}$ such that $s_{1}=s$ and

(1) in the divided enrollment system, each student in the list receives the unified enrollment school of the preceding student, i.e., for each $i \in\{2, \ldots, n\}, \mu^{d}\left(s_{i}\right)=$ $\mu^{u}\left(s_{i-1}\right)$, and student $s_{1}$ receives the unified enrollment school of student $s_{n}$, i.e., $\mu^{d}\left(s_{1}\right)=\mu^{u}\left(s_{n}\right)$,

(2) the school that each student receives in the unified enrollment system does not have any empty seats in the divided enrolment outcome, i.e., for each $i \in\{1, \ldots, n\}$, if $\mu^{u}\left(s_{i}\right)=c$, then $\left|\mu^{d}(c)\right|=q_{c}$,

(3) the unified enrollment schools of all the students in the list are of the same type, and

(4) student $s^{*}$ is an interrupter for a student $s^{\prime} \in\left\{s_{1}, \ldots, s_{n}\right\}$ at the unified enrollment school of $s^{\prime}$.

Theorem 3. Suppose that choice rules of schools satisfy substitutability and conditional capacityfilling. Then each student whose unified and divided enrollment schools are different either suffers from waste, miscoordination, or a new interrupter.

\section{Conclusion}

As more student assignment programs are being adapted by school districts, policymakers face the challenge of implementing enrollment systems. The Chicago Board of Education has taken a big step in this direction to implement a centralized clearinghouse for student assignment and it has decided to implement a divided enrollment system. Even though this system is easy to use by parents because it is based on the student-proposing 
deferred acceptance algorithm (SPDA) which is strategy-proof, it can produce an outcome that is not stable. More importantly, we have shown that the alternative unified enrollment system, which produces the student-optimal stable matching, is weakly better for all students than the divided enrollment system when choice rules are substitutable. We have also identified three sources of inefficiency that characterize the entire efficiency loss due to the divided enrollment system. Our analysis has shown that seemingly natural student assignment mechanisms may have serious deficiencies even when they are based on well-behaved algorithms such as SPDA. However, market-design research can guide policymakers achieve the desirable policy goals in a centralized clearinghouse.

\section{References}

Abdulkadiroğlu, Atila and Tayfun Sönmez, "School choice: A mechanism design approach," American Economic Review, June 2003, 93 (3), 729-747.

, Parag Pathak, Alvin Roth, and Tayfun Sönmez, "The Boston Public School Match," American Economic Review, May 2005, 95 (2), 368-371.

Abdulkadiroğlu, Atilla, Parag A. Pathak, and Alvin E. Roth, "The New York City High School Match," American Economic Review, 2005, 95 (2), pp. 364-367.

Aygün, Orhan and Tayfun Sönmez, "Matching with Contracts: Comment," American Economic Review, 2013, 103 (5), 2050-2051.

Bonkoungou, Somouaoga, "University Admissions Reform in France: An Analysis of a New Mechanism," 2017. Working Paper.

Chambers, Christopher P. and M. Bumin Yenmez, "A simple characterization of responsive choice," 2017. Working paper.

Dubins, Lester E and David A Freedman, "Machiavelli and the Gale-Shapley algorithm," The American Mathematical Monthly, 1981, 88 (7), 485-494.

Echenique, Federico, "Counting combinatorial choice rules," Games and Economic Behavior, 2007,58 (2), $231-245$.

and M. Bumin Yenmez, "How to Control Controlled School Choice," American Economic Review, August 2015, 105 (8), 2679-2694.

Gale, David and Lloyd S. Shapley, "College Admissions and the Stability of Marriage," The American Mathematical Monthly, January 1962, 69 (1), 9-15.

Haeringer, Guillaume and Vincent Iehlé, "Gradual college admission," 2017. Working Paper.

Hatfield, John William and Paul R. Milgrom, "Matching with Contracts," American Economic Review, September 2005, 95 (4), 913-935.

Kesten, Onur, "School choice with consent," The Quarterly Journal of Economics, 2010, 125 (3), 1297-1348. 
Manjunath, Vikram and Bertan Turhan, "Two school systems, one district: What to do when a unified admissions process is impossible," Games and Economic Behavior, 2016, 95, $25-40$.

Roth, Alvin E., "The Economics of Matching: Stability and Incentives," Mathematics of Operations Research, 1982, 7, 617-628.

Turhan, Bertan, "Welfare and Incentives in Partitioned School Choice Markets," 2017. Working Paper.

Wu, Qingyun and Alvin E. Roth, "The lattice of envy-free matchings," Games and Economic Behavior, 2018, 109, $201-211$.

\section{Appendix A. Proofs}

In this appendix, we include the omitted proofs.

Proof of Lemma 1. Without loss of generality, assume that $c \in \mathcal{C}^{1}$. Let $S^{t}(c)$ be the set of students who have applied to school $c$ at or before Step $t$ in the unified enrollment system. Similarly, let $\tilde{S}^{t}(c)$ be the set of students who have applied to school $c$ at or before Step $t$ in the divided enrollment system. We want to show that $S^{t}(c) \subseteq \tilde{S}^{t}(c)$ for every type $t$. We prove this claim by mathematical induction on $t$.

When $t=1$, each student applies to his top overall ranked school in the unified enrollment system. Therefore, the set of applicants in the unified enrollment system to school $c$ is $S^{1}(c)=\left\{s \mid c P_{s} c^{\prime}\right.$ for every $c^{\prime} \in \mathcal{C} \cup\{s\}$ such that $\left.c^{\prime} \neq c\right\}$. On the other hand, in the divided enrollment system, each student applies to the top-ranked school for every type of school. That is, $\tilde{S}^{1}(c)=\left\{s \mid c P_{s} c^{\prime}\right.$ for every $c^{\prime} \in \mathcal{C}^{1} \cup\{s\}$ such that $\left.c^{\prime} \neq c\right\}$. Clearly, $S^{1}(c) \subseteq \tilde{S}^{1}(c)$.

Assume that the claim holds for every number smaller than $t$. We now show it for $t \geq 2$. Let $s$ be a student who has applied to school $c$ at Step $t$ in the unified enrollment system, that is, $s \in S^{t}(c) \backslash S^{t-1}(c)$. We claim that $s \in \tilde{S}^{t}(c)$. If school $c$ is the highest-ranked school among $\mathcal{C}^{1}$ with respect to $P_{s}$, then $s \in \tilde{S}^{1}(c)$, which implies $s \in \tilde{S}^{t}(\mathrm{c})$. Otherwise, if school $c$ is not the highest-ranked school, there exists a school $c^{\prime} \in \mathcal{C}^{1}$ which is ranked right above $c$ among schools in $\mathcal{C}^{1}$ with respect to $P_{s}$. Since $s \in S^{t}(c) \backslash S^{t-1}(c)$, school $c^{\prime}$ must have rejected student $s$ at Step $t-1$ or before in the unified enrollment system, that is $s \in S^{t-1}\left(c^{\prime}\right)$ and $s \notin C h_{c^{\prime}}\left(S^{t-1}\left(c^{\prime}\right)\right)$. By the mathematical induction assumption, $s \in \tilde{S}^{t-1}\left(c^{\prime}\right)$ and by substitutability $s \notin C h_{c^{\prime}}\left(\tilde{S}^{t-1}\left(c^{\prime}\right)\right)$ because $\tilde{S}^{t-1}\left(c^{\prime}\right) \supseteq S^{t-1}\left(c^{\prime}\right)$ and $s \notin C h_{c^{\prime}}\left(S^{t-1}\left(c^{\prime}\right)\right)$. Therefore, student $s$ applies to school $c$ at Step $t$ or before in the divided enrollment system, so $s \in \widetilde{S}^{t}(c)$.

We have shown that every student in $S^{t}(c) \backslash S^{t-1}(c)$, is also in $\tilde{S}^{t}(c)$. This and the mathematical induction hypothesis that $S^{t-1}(c) \subseteq \tilde{S}^{t-1}(c)$ imply $S^{t}(c) \subseteq \tilde{S}^{t}(c)$. 
Proof of Theorem 1. SPDA ends in a finite number of steps under both systems. Let $\mu$ be the matching produced by the unified enrollment system and $\tilde{\mu}_{k}$ be the matching produced by type $k$ in the divided enrollment system. Then the outcome for student $s$ in the divided enrollment system is $\tilde{\mu}(s) \equiv \max _{P_{s}}\left\{\tilde{\mu}_{1}(s), \ldots, \tilde{\mu}_{K}(s)\right\}$. Assume, for contradiction, that $c \equiv \tilde{\mu}(s) P_{s} \mu(s)$.

Let $t$ be bigger than the number of steps in SPDA under both systems and $S^{t}(c)$ be the set of students who have applied to school $c$ at or before Step $t$ in the unified enrollment system. Similarly, let $\tilde{S}^{t}(c)$ be the set of students who have applied to school $c$ at or before Step $t$ in the divided enrollment system. Since $c=\tilde{\mu}(s), s \in C h_{c}\left(\tilde{S}^{t}(c)\right)$. Furthermore, since $c P_{s} \mu(s)$, student $s$ must have been rejected by school $c$ in the unified enrollment system. Therefore, $s \in S^{t}(c)$. But student $s$ is not assigned to school $c$ in the unified enrollment system, so $s \notin C h_{c}\left(S^{t}(c)\right)$. Lemma 1 shows that the set of applicants in the unified system is a subset of the set of applicants in the divided enrollment system for any step, so $S^{t}(c) \subseteq \tilde{S}^{t}(c)$. This fact together with $s \notin C h_{c}\left(S^{t}(c)\right)$ and $s \in C h_{c}\left(\tilde{S}^{t}(c)\right)$ yield a contradiction because $C h_{c}$ is substitutable.

Proof of Theorem 2. Suppose that the choice rule of school $c$ does not satisfy substitutability. Then there exist students $s$ and $s^{\prime}$, and a set of students $S$ with $s \neq s^{\prime}$ and $S \cap\left\{s, s^{\prime}\right\}=\varnothing$ such that $s \in C h_{c}\left(S \cup\left\{s, s^{\prime}\right\}\right)$ and $s \notin C h_{c}(S \cup\{s\})$.

Consider the following student preferences. For each student in $S \cup\{s\}$, school $c$ is the only acceptable school. For student $s^{\prime}$, the most preferred school has a different type than the type of school $\mathrm{c}$ and the second-most preferred school is $c$. There are no other acceptable schools for student $s^{\prime}$. The remaining students do not have any acceptable schools.

In the unified enrollment system, at Step 1, students in $S \cup\{s\}$ apply to school $c$ and student $s^{\prime}$ applies to another school. There are no other applications. School $c$ tentatively accepts students in $C h_{c}(S \cup\{s\})$ and rejects students in $(S \cup\{s\}) \backslash C h_{c}(S \cup\{s\})$. By assumption, student $s$ is rejected. Since there are no more acceptable schools for student $s$, she is unmateched in the unified enrollment system.

In the divided enrollment system, at Step 1, students in $S \cup\left\{s, s^{\prime}\right\}$ apply to school $c$. School $c$ tentatively accepts $C h_{c}\left(S \cup\left\{s, s^{\prime}\right\}\right)$, which includes student $s$. For the rejected students, there is no remaining acceptable school that has the same type with school $c$. Therefore, student $s$ is matched with school $c$ in the divided system.

Student $s$ strictly prefers the divided enrollment outcome to the unified enrollment outcome.

Proof of Theorem 3. Recall that $S$ is the set of students who are matched to different schools at the truthful equilibrium of the divided enrollment system and the outcome of the unified 
enrollment system. Suppose that $s \in S$, i.e., $\mu^{u}(s) \neq \mu^{d}(s)$, and also that student $s$ does not suffer from waste. Note that by substitutability Theorem 1 applies, and therefore student $s$ strictly prefers $\mu^{u}(s)$ to $\mu^{d}(s)$. Then $\mu^{u}(s)$ must be a school since $\mu^{d}(s)$ is individually rational for student $s$. Consider the set $S^{\prime} \subseteq \mathcal{S}$ of students constructed by the following algorithm. Set $S^{\prime}=\varnothing$ initially.

In Step 1, let $S_{1}^{\prime}$ denote the set of students who are assigned to $\mu^{u}(s)$ at the divided enrollment outcome, while they were assigned to a different school at the unified enrollment outcome, i.e., $S_{1}^{\prime}=\left\{s^{\prime} \in S: \mu^{d}\left(s^{\prime}\right)=\mu^{u}(s)\right\}$. Update $S^{\prime}=S_{1}^{\prime}$.

In Step $n>1$, let $S_{n}^{\prime}$ denote the set of students such that for each $s^{\prime} \in S_{n}^{\prime}$, there exists $s^{\prime \prime} \in S_{n-1}^{\prime}$ such that $s^{\prime}$ is assigned to $\mu^{u}\left(s^{\prime \prime}\right)$ at the at the divided enrollment outcome, while $s^{\prime}$ is assigned to a different school at the unified enrollment outcome, i.e., $S_{n}^{\prime}=\left\{s^{\prime} \in S\right.$ : $\exists s^{\prime \prime} \in S_{n-1}^{\prime}$ such that $\left.\mu^{d}\left(s^{\prime}\right)=\mu^{u}\left(s^{\prime \prime}\right)\right\}$. Update $S^{\prime}=S_{n}^{\prime} \cup S_{n-1}^{\prime}$.

Since there are finitely many students, there exists a step $M$ such that $S_{M}^{\prime}=\varnothing$ and $S^{\prime}$ is constant after step $M$, where we stop the algorithm.

We claim that $s \in S^{\prime}$. Since student $s$ does not suffer from waste, for each $s^{\prime} \in S^{\prime} \cup\{s\}$, the capacity of $\mu^{u}\left(s^{\prime}\right)$ must be full at the divided enrollment outcome. Let $C=\{c \in \mathcal{C}$ : $\exists s^{\prime} \in S^{\prime} \cup\{s\}$ such that $\left.\mu^{u}\left(s^{\prime}\right)=c\right\}$ be the set of unified enrollment schools of $S^{\prime} \cup\{s\}$. For each $c \in C$, let $H(c)$ denote the set of students who are not assigned to school $c$ at the unified enrollment but are assigned to school $c$ at the divided enrollment. Since for each $s^{\prime} \in S^{\prime} \cup\{s\}$, the capacity of $\mu^{u}\left(s^{\prime}\right)$ is full at the divided enrollment outcome and $\mu^{u}\left(s^{\prime}\right) \neq$ $\mu^{d}\left(s^{\prime}\right)$, we have $\left|\cup_{c \in C} H(c)\right| \geq\left|S^{\prime} \cup\{s\}\right|$. But also, by construction $\cup_{c \in C} H(c)=S^{\prime}$, which implies that $s \in S^{\prime}$.

Since $s \in S^{\prime}$, there exists a list of students, including student $s$, satisfying the first two properties described in the definition of miscoordination. If there exists a student in the list whose unified enrollment school belongs to a different type than the unified enrollment school of another student in the list, then student $s$ suffers from miscoordination. Otherwise, the list satisfies the properties 1,2, and 3 in the definition of a new interrupter. Note that the unified enrollment schools of all the students in the list are of the same type.

Without loss of generality, suppose that the unified enrollment schools of all the students in the list are selective. Let $t$ be the first step in SPDA for selective schools in the divided enrollment system where a student in the list, say student $s^{\prime}$, is rejected by his unified enrollment school, say school $c$. Note that $s^{\prime}$ is acceptable for school $c$, and by conditional capacity-filling, the capacity of $c$ is full at the end of Step $t$. Note that the student succeeding student $s^{\prime}$ in the list receives school $c$ in the divided enrollment system, and moreover, he applies to and gets accepted by school $c$ after Step $t$, since $t$ is the first step in SPDA for selective schools in the divided enrollment system where a student in the list is rejected by his unified enrollment school. But then, there exists a student $s^{*}$ who is temporarily 
accepted by school $c$ at Step $t$ and is rejected by school $c$ after Step $t$. But then, student $s^{*}$ is an interrupter for $s^{\prime}$ at school $c$. Thus, student $s$ suffers from a new interrupter. 